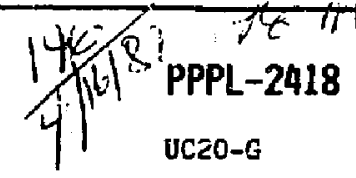

UC20-G

\section{(3)}

$-7-30 / 69$

PPPL-2418
PPPI -2418

DE87 008070

\title{
MICROINSTABILITY-BASED MUDELS FOR CONFINEMENT PROPERTIES AND IGNITION CRITERIA IN TOKAMAKS
}
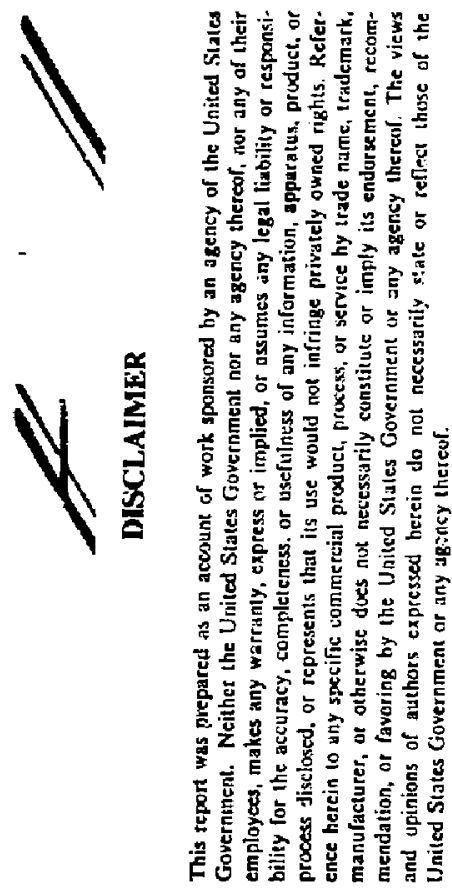

By

W.M. Tang, C.H. Blshop, B. Copp1, S.M. Kaye, F.H. Perkins, M.H. Redi, and G. Rewoldt

\section{PLASMA PHYSICS LABORATORY}

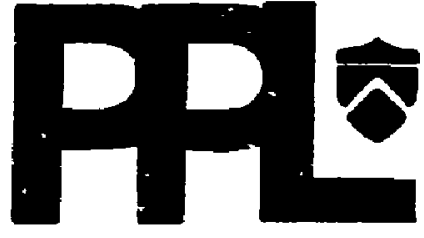

PRINCETON UNIVERSITY PRINCETON, NEW JERSEY

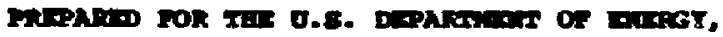

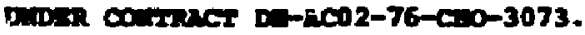




\section{MICROINSTABILITY-BASED MODELS FOR CONFINEMENT PROPERTIES AND IGNITION CAITERIA IN TOKAMAKS}

W. M. TANG, C. M. BISHOP", B. COPPI"*, S. M. KAYE, F. W. PERKINS, M. H. REDI, AND G. REWOLDT

Plasma Physics Laboratory, Princeton University P.0. Box 451, Princeton, New Jersey, USA

\section{ABSTRACT}

This paper reports on results of theoretical studies dealing with: (1) the use of microinstability-based thermal transport models to interpret the anomalous confinement properties observed in key tokamak experiments such as TFTR and (2) the likely consequences of the presence of such instabilities for future ignition devices. Transport code simulations using profile-consistent forms of anomalous thermal diffusivities due to drift-type instabilities have yielded good agreement with the confinement times and temperatures observed in TFTR under a large variety of operating conditions including pellet-ruelling in both ohmic-and neutral-beam-heated discharges. With regard to achieving an optimal ignition margin, the adverse temperature scaling of anomalous losses caused by drift modes leads to the conclusion that it is best to operate at the maximum allowable density while holding the temperature close to the minimum value required for ignition.

\footnotetext{
* Permanent Address: Culham Laboratory, UK

Permanent Address: Massachusetts Institute of Technology, Cambridge, MA, USA
}

\section{MASTE}


I. ANALYSIS OF ANOMALOUS THERMAL CONFINEMENT IN TOKAMAKS

Identification of the primaty physical processes responsible for the anomalous thermal transport properties currently observed in tokamaks is vitally important not only for the effective development of methods to improve performance in existing devices but also for the proper planning of future ignition experiments. In recent work [1-4] it has been demonstrated that if realistic profile constraints are invoked, then most of the significant confinement trends in low-beta tokamaks can be explained by electrostatic $\left(E \times \underline{B}_{0}\right)$ transport caused by drift-type microinstabilities. Anomalous thermal transport models based on the associated physics have yielded predictions in very favorable agreement with a substantial experimental data base with respect to both the scaling and the magnitude of the energy confinement time $\left(\tau_{\mathrm{E}}\right)$ and the central electron temperature ( $\mathrm{T}_{\mathrm{eo}}$ ).

In the present studies, the profile-consistent microinstability models derived in Ref. 2 have been used in the BALDUR ( $t$ ime-dependent, 1-D) transport code [5] for the purpose of interpreting experimental results from TFTh including ohmic heating, neutral-beam-injection (NBI) heating, and pelletfuelling in both onmic- and NBI-heated discharges. The theoretical expressions for the anomalous thermal diffusivities $\left(x_{e}, x_{i}\right)$ are based on the presence of trapped-electron drift modes and/or toroidal ion temperature gradient $\left(\eta_{1}\right)$ modes in the bulk region of optimal thermal insulation (roughly located between the $q=1$ and $q=2$ surfaces in the plasma). They incorporate the familiar mixing length estimate for the magnitude of the fluctuations and also include the assumption that a temperature "profile consistency" constraint [6] is satisfied. The latter is required here becsuse the present state of development of nonlinear toroidal microinstability theories cannot provide reliable scaling with non-dimensional parameter-s such as $q$ and because 
mechanisms outside the realm of microinstabilities could be playing a prominent role outsige the $q=2$ region. If the parameter, $\eta_{i} \equiv d e n T_{i} / d e n n_{i}$, remains below a critical magnitude, $\left(n_{i}\right)_{c}$ (roughly about 2 ), then the dominant instability is the trapped-electron mode, and the associated form of the electron diffusivity can be expressed as

$$
x_{e}^{T E}\left(\mathrm{~cm}^{2} / \mathrm{sec}\right)=\frac{0.4\left(1+.25 a_{n}\right) 10^{4} e^{0.8_{E_{H}}}(r)}{n_{e 0^{R}}{ }^{1.2} a^{0.1} z^{0.2_{B_{T}} 0.4_{q_{a}} 0.9}}
$$

with

$$
F_{H}(r)=\frac{\left[1-\exp \left(-a_{a}\right)\right] \exp \left(2 a_{g} r^{2} / 3 a^{2}\right) \int_{0}^{r / a} d x x h(x)}{\left[n_{e}(r) / n_{e 0}\right]\left(r^{2} / a^{2}\right) \int_{0}^{1} d x x h(x)},
$$

where $a_{q}=q_{a}+0.5, P(M W)$ is the total equilibrated ("steady-state") value of tile input power (minus radiation losses); $h(x)$ is the energy deposition profile, $r_{e 0}\left(10^{14} \mathrm{~cm}^{-3}\right), R(\mathrm{~m}), a(m), B_{T}(T)$, and $a_{n}$ is the usual parabolic density profile parameter. In arriving at $\mathrm{Eq} .(1)$, the intrinsic temperature dependence has been eliminated in terms of a power dependence through an assumed thermal steady state [2]. The influence of these modes on $x_{i}$ tends to be masked by the usual ion neoclassical transport [2]. However, if $n_{1}$, $\left\langle\eta_{i}\right\rangle_{c}$, then the toroidal $\eta_{i}$-modes become cominant $[7]$, and the associated profile-consistent form of the anomalous ion diffusivity becomes

$$
x_{i} n_{i}(r)=\frac{10^{3}\left(p / n_{e o}\right)^{0.6}}{\left(R B_{T} q_{a}\right)^{0.8_{(a)}} F^{0.2}} F_{H}(r) .
$$

For a surficiently collisionless plasma, $x_{e}$ also scales as Eq. (2). On the other hand, for values of $v_{*}$ (the usual banana regime collisionality parameter) $\geqslant 0.1$ to 0.2 , the electron loss channel remains sensitive to 
collisions in the presence of $\eta_{\mathrm{i}}$-mades. This is due to the fact that even though it does not strongly affect these instabilities, the non-adiaid: $z$ trapped-electron response does determine $x_{e}$. Hence, the associated form of $x_{e}$ tends to be very similar to Eq. (1).

Simulations of nearly two dnzen TFTR discharges covering a wide range of experimental conditions were carried out with the BALDUR transport code using $x_{e}=x_{e}^{N E O}+x_{e}^{T E}, x_{i}=x_{i}^{N E O}+x_{i}^{n_{i}}$ (with $x_{i}^{\eta_{i}}=0$ if $\eta_{i} \leq 1.5$ at $q=1.5$ ), Other important effects such as sawteeth behavior inside the $q=1$ region, radiation processes, etc. have also been included and are described in Ref. 5 . With regard to particle transport, empirical models were constructed to produce density profiles approximating the Thomson-scattering-measured density data. In particular, $D=D^{N E O}+D^{A N O M}$ and $v=v^{N E O}+v^{A N O M}$. For example, in simulating the pellet-fuelled cases, $D^{\text {ANOM }}\left(\mathrm{cm}^{2} / \mathrm{sec}\right)=4 \times 10^{16 / \mathrm{n}}$ (r) with $v^{A N O M}=2 D^{\text {ANOM }_{r} / a^{2}}$ before and $v^{\text {ANOM }}=0$ after the pellets are injected. The results from the predictive simulations of numerous TFTR large plasma discharges are summarized on Fig. 1 and Table 1. On Fig. 1 the calculated energy confinement times are plotted as a function of the familiar neo-Alcator parameter and compared with the experimental results from a large number of gas-fuelled ohmically heated discharges (crosses) as well as from pelletfuelled ohmic cases (open diamonds). For the gas-fuelled cases it is quite clear that the anomalous enhancement of $x_{i}$ associated with $n_{i}$-modes (solid circles) can account for the observed saturation of ${ }^{\tau} E$ at values below those predicted by calculations using only $:_{i}^{N E O}$ (open circles). The result from the simulation of an ohmic PDX plasma is also displayed to indicate consistent size-scaling at a small value of the neo-Alcator parameter. With regard to the pellet-fuelled cases the simulation results are again in very good agreement with the experimental data. As shown on Fig. 1, the first pair of 
diamonds corresponds to values of $\mathrm{r}_{\mathrm{E}}$ at two points in time after the injection of a single pellet, and the second pair of diamonds to the analogous situation for a three-pellet experiment. The higher $\tau_{E}$ in each pair represents the confinement at the time closer to the time of injection. In these cases the global confinement times are lower than those for the gas-fuelled experiments because of larger energy losses associated with convection and radiation. However, the simulations also indicate that the central confinement is significantly improved after pellet infection because of the resultant peaking in the density profile. This trend, which is in agreement with that found in the TRANSP data analysis, is likely due to $x_{e}$ scaling as $1 / n_{e}(r)$ and to the fact that the $\eta_{i}$-driven anomalous enhancement of $x_{i}$ can be eliminated (e.s., in the 3-pellet case). Results for $\tau_{E}$ and $T_{e}$ from simulations of a representative set of NBI-heated TFTR discharges are given in Table 1 and show good agreenent with the experimental values. These include a pellet-injection NBI case (shot 14773) and a typical "supershot" discharge (shot 22014). All of the results displayed in Fig. 1 and Table 1 were computed using Eq. (1). With regard to the supershot case, prelininary calculations using a more realistic version of $x_{e}(r)$ (which takes into account only the power into the electron channel and the proper transition into the collisionless regime) has yielded olose agreement with the experimental values given in Table 1; i.e., $\tau_{E}=0.14 \mathrm{~s}$ and $T_{e}=5.2 \mathrm{keV}$. In addition, all of the computed temperature profiles correlated well with the data, and comparisons of the theoretical $x_{e}(r)$ given by $\mathrm{Eq}$. (1) and those obtained from TRANSP data analysis studies of a number of ohmic- and NBI-heated IFTR discharges have also indicated reasonable agreement.

Before proceeding with the further development and application of the profile-consistent models just described to the important class of H-mode NBI- 
heated divertor plasmas, it is necessary to first establish the proper behavior of the microinstabilities invoked under such conditions. In previous studies involving comprehensive kinetic toroidal computations [8], it was demonstrated that the weaker pressure gradients in the interior region of $\mathrm{H}-$ mode plasmas led to correspondingly weaker instabilities. Altholigh this reduction in the lonal anomalous thermal diffusivity in the inner zone is consistent with the observed improvement in $\tau_{E}$, it leaves open the question of the nature of the transport froperties in the very steep gradient region near the edge. Specifically, in order to be consistent with the thermal fluxes calculated in the interior, the local thermal diffusivity in the edge zone must be greatly rediced. This issue has been addressed in tne present work by interfacing the kinetic stability code [8] with a model tokamak equilibrium with a separatrix [9]. The equilibrium here takes into account the enhanced local shear effects near the separatrix and is basically an extension of the familias "s- $\alpha$ model" [10] whose rinite-beta properties are primarily gaverned by the magnitude of the local pressure gradient. Fully electromagnetic salculations of the dominant toroidal microinstabilities were caried out using as input parameters the edge data obtained from careful measurements of H-mode and L-mode PDX tokamair discharges. The results generally indicate that the proximity of the separatrix to the magnetic surface considered has a significant stabilizing influence. This effect is considerably stronger for the H-mode cases with their characteristically steeper edge profiles. In particular, the finite-beta-modified drift-type electrostatio modes are found to be stable over a regicn extending several centimsters inside the separatrix for the H-mode profiles but over a much smaIler range for the L-inode. These enhanced local stability properties neas the separatrix thus serve to support the notion that thermal transport properties in if-mode plasmas are 
reconcilable with microinstability-based models.

\section{CONSEQUENCES OF MICROINSTABILITIES FOR IGNITION IN TOKAMAKS}

The favorable correlations between the confinement trends observed under a large variety of tokamak operating conditions and the results from the anomalous transport studies just described as well as from previous work [1-4] serve to emphasize the importance of addressing the consequences of drift-type instabilities for future ignition devices. Under steady-state conditions, the simplest form of the basic homogencous \{zero-dimensional\} thermal energy balance equation is given by

$$
P_{h}=P_{L e}+P_{L i}+P_{R}=P_{a} \text {, }
$$

whore $P_{h}$ is the external heating power density, $P_{L e}$ and $P_{L i}$ represent the losses due to electron and and ion conductivity, $P_{R}$ represents radiation losies, and $P_{a}$ is the a-particle heating term. in earlier calculations $\{11,12]$ it was demonstrated that the familiar Murakami density limit represents a balance between radiation losses $\left(P_{R}\right)$ and ohmic heating input power $\left(P_{h}\right)$. However, since the fusion power $\left(P_{\alpha}\right)$ will offset $P_{R}$ at temperatures characteristic of ignition conditions $\left(T_{0}>6\right.$ keV), this limit should not be relevant. Instead, attention needs to be focused on the influence of enhanced forms of $P_{L e}$ and $P_{L_{i}}$ caused by the presence of microinstabilities. First consider the situation where the dominant terms in Eq. (1) are $P_{\alpha}, P_{R}$ (Erom bremsstrahlung), and $P_{\text {Le }}$ from aromalous losses associated with the dissipative trapped-electron modes. Using the simple local form for $x_{e}$ given in Ref. 1 and applying the boundary conditions of sawtooth flattening for $q \leq 1$ and $T_{e}=0$ for $q \geq 2$, the corresponding 1-D 
radial equation yields eigenvalıe solutions which can be expressed in the form of an ignition criterion,

$$
\left[n_{0}\left(10^{15} \mathrm{~cm}^{-3} \mathrm{JB}_{\mathrm{T}}(10 \mathrm{~T})\right]^{2}[\mathrm{a}(\mathrm{m})]^{2.5}[\mathrm{R}(\mathrm{m})]^{1.5}>14.6\left(3 / \mathrm{q}_{\mathrm{a}}\right)^{1.5} \mathrm{a}_{\mathrm{n}}^{2}\right.
$$

Since $P_{L e}$ is independent of density in the dissipative trapped-electron regime, it can be concluded that for ignition experiments falling in this regime, the density can be increased to the point where the criterion given by Eq. (4) can be satisfied, provided external power $\left(P_{h}\right)$ is supplied to compensate for the heat capacity of the increased density and to maintain $\mathrm{T}_{o}$ > $6 \mathrm{keV}$ so that $P_{a}>P_{R}$. The power required to balance the thermal conduction losses is estimated to be $P_{\text {crit }}(M W)=120\left(3 / \mathrm{q}_{a}\right)^{2.75}\left(\mathrm{~T}_{\mathrm{o}} / 6 \mathrm{keV}\right)^{4.5}$ $a_{n}^{2}\left(\left\langle 2 B_{T}{ }^{2}(a R)^{0.5}\right)\right.$. These results clearly suggest that the optimal ignition scenario would involve operating at (i) the lowest allowable temperature (consistent with ignition), (ii) the highest allowable dersity (consistent with MHD B-limits but not the Murakami limit), and (iii) the flattest density profiles (because of the strong $a_{n}$-dependence in the local model used for $x_{e}$ ). However, with regard to (ij.i), it should be strongly cautioned that if the density profiles are allowed to be too flat, then $n_{i}$ can easily exceed $\left(n_{i}\right)_{e^{-}}$The consequent enhancement of $P_{L i}$ due to $\eta_{i}$-instabilities can then pose a very restrictive density constraint in the ignition temperature regime. In order to simply illustrate this point, it is corvenient to express Eq. (3) in a schematic form highlighting the density and temperature dependences in each term, i.e., (taking $T_{I}=T_{e}=T$ ).

$$
P_{h}-r_{T E} T^{9 / 2}=n^{2}\left({ }_{Y_{B N}} T^{1 / 2}-\gamma_{a} T^{5 / 2}\right)+n \gamma_{\eta_{i}} T^{5 / 2}
$$


with. ${ }_{\mathrm{TE}} \mathrm{T}^{9 / 2}$ being the dissipative trapped-electron losses, $\mathrm{n}^{2} \mathrm{~T}^{1 / 2}{ }_{\mathrm{BN}}$ being losses due to bremsstrahlung and ion neaclassical conduction, $Y_{\alpha} T^{5 / 2} \equiv P_{a}$, and $n r_{n_{i}} T^{5 / 2}$ being the $n_{i}$-mode losses. In the absence of the last term, Eq. (5) clearly indicates that provided $\mathrm{P}_{h}>{ }_{\mathrm{TE}} \mathrm{T}^{9 / 2}$, there wolld indeed be no density limit for $T \geq T_{M I}$ with $T_{M I} \equiv\left(\gamma_{B N} / \gamma_{\alpha}\right)^{1 / 2} \equiv$ minimum ignition temperature. However, if present, this density-dependent loss term would force operating beiow an upper density bound $a t T \leq T_{M I}$ and above a lower density bound at $T>$ $T_{M I}$. The latter situation simply corresponds to requiring $P_{\alpha}>n_{Y_{\eta_{j}}} T^{5 / 2}$. Estimates from Eq. (5) indicate that the external power needed to reach ignition is proportional to $\mathrm{T}_{M T}{ }^{9 / 2}$. Hence, in order to avoid such stringent constraints, pellet-injection-fuelling procedures could be adopted to produce density profiles sufficiently peaked to suppress the $n_{i}$-modes. After exceeding tne required ignition temperature, a less discriminating fuelling system would be adequate since $P_{\alpha}$ could then offset any ensuing anomalous losses of this type. In general, for $T>T_{m I}$, the power losses resulting from drift mortes increase at least as rapidly with $T$ as the fusion power. Consequently, the optimum path to ignition should exploit favorable scalings with $B_{T}$, density, size, etc., while holding $T$ close to $T_{m I}$. 
ACKNOWLEDGMENTS

We are indebted to P.C. Efthimion, G.L. Sohmidt, D.R. Mikiselsen, and the TETR physicists for providing the deta as well as vaiuati: assiztance needed to carry out the BALOUR Simuiations. Special thanks are extended to 5. Hiroe, P. LeBIanc, and B. Grek for poviding the profile data from fox used in the H.mode calculaticns.

This work was supported by United States Department of Energy Contract No. DE-ACO2-76-CHO-3073. F. W. Perkins was also supported by NASA Contract No. NAGW-9! and by the Nationa! Bureau of Standards. 
REEERENCES

[1] PERKINS, F.W., Proc. 4th Int. Symp. on Heating in Toroidal Plasmas, Rome, 1984, Vol. 2, p. 977; PEAKINS, F.U., SUN, Y.C., Princeton Plssma Physies Laboratory Report No. PPPL-2261 (1985).

[2] TANG, W.M., Princeton Plasma Physics Laboratory Report No. PPPL-2311 (1986), Nucl. Eusion (in press).

[3] DOMINGUEZ, R.R., WALTZ, R.E., GA Technologies Rep. GA-18184 (1986).

[4] fomanelli, F., TANG, W.M., WHITE, R.B., Princeton Plasma Physios Laboratory Report No. PPPL-2310 (i986), Nucl. Fusion (in press).

[5] SINGER, C.E., et al., Princeton Plasma Physics Laboratory Report No. PPPL-2073 (1986).

[6] COPPI, B., Comments Plasma Phys. Cont. Fusion $\underline{5}, 261$ (1980).

[7] TANG, W.M., REWOLDT, G., CHEN, L., Princeton Plasma Physics Laboratory Report No. PPPL-2337 (1986), Phys. Fluids (in press).

[8] TANG, W.M., et al., Nucl. Eusion 25, 151 (i985); REWOLDT, G., et al., Phys. Fluids $\underline{25}, 480$ (1982).

[9] BISHOP, C.M., Culham Laboratory Rep. CLM-P764 (1985).

[10] CONNOR, J.H., et al., Phys. Rev. Lett. 40, 396 (1978).

[11] PERKINS, F. H., HULSE, R. A., Phys. Fluids 28, 1837 (1985).

[12] COPPI, B., TANG, H. M., Princeton Plasma Physics Laboratory Report No. PPPL-2343 (1986). 
Table 1. Parameterg of Simulated Neutral-Beam-

Heated TFIr shots

\begin{tabular}{|c|c|c|c|c|c|c|c|c|c|c|c|c|c|}
\hline Shot & $I_{p}^{(M A)}$ & $\mathrm{B}_{\mathrm{Z}}^{(\mathrm{T})}$ & $\mathrm{R}^{(\pi)}$ & $A^{(n x)} \bar{n}$ & $\tilde{n}_{e}\left(10^{19} m^{3}\right)$ & $P_{i, n j}(M K)$ & $z_{\text {eff }}$ & $\mathrm{q}_{a}$ & $T_{e}^{\exp }(k \in V)$ & $\mathrm{r}_{e}^{\mathrm{sim}}$ & $\tau_{\mathrm{E}}^{\exp (s)}$ & $\tau_{\mathrm{E}}^{\mathrm{Sim}}$ & $v_{t e}^{l q=1.5}$ \\
\hline 14727 & 2.2 & 4.8 & 2.56 & 0.82 & 4.8 & 5.3 & 2.6 & 2.8 & 4.0 & 3.6 & 0.20 & 0.18 & 0.19 \\
\hline 14773 & 2.2 & 4.7 & 2.57 & 0.82 & $3.0\left(7.0^{*}\right)$ & 5.7 & 1,9 & 2.8 & 2.4 & 2.8 & 0.14 & 0.19 & 0.29 \\
\hline 19965 & 2.2 & 4.8 & 2.49 & 0.83 & 4.2 & 8.8 & 3.2 & 3.0 & 3.8 & 3.7 & 0.11 & 0.11 & 0.25 \\
\hline \multirow[t]{2}{*}{22014} & 0.8 & 4.6 & 2.55 & 0.82 & 3.2 & 11.3 & 2.5 & 7.6 & 5.8 & 3.5 & 0.12 & 0.06 & 0.13 \\
\hline & & & & & & & & & & $6.2 t$ & & $0.14+$ & \\
\hline
\end{tabular}

*After Pellét'Injection

tCollisional Model 


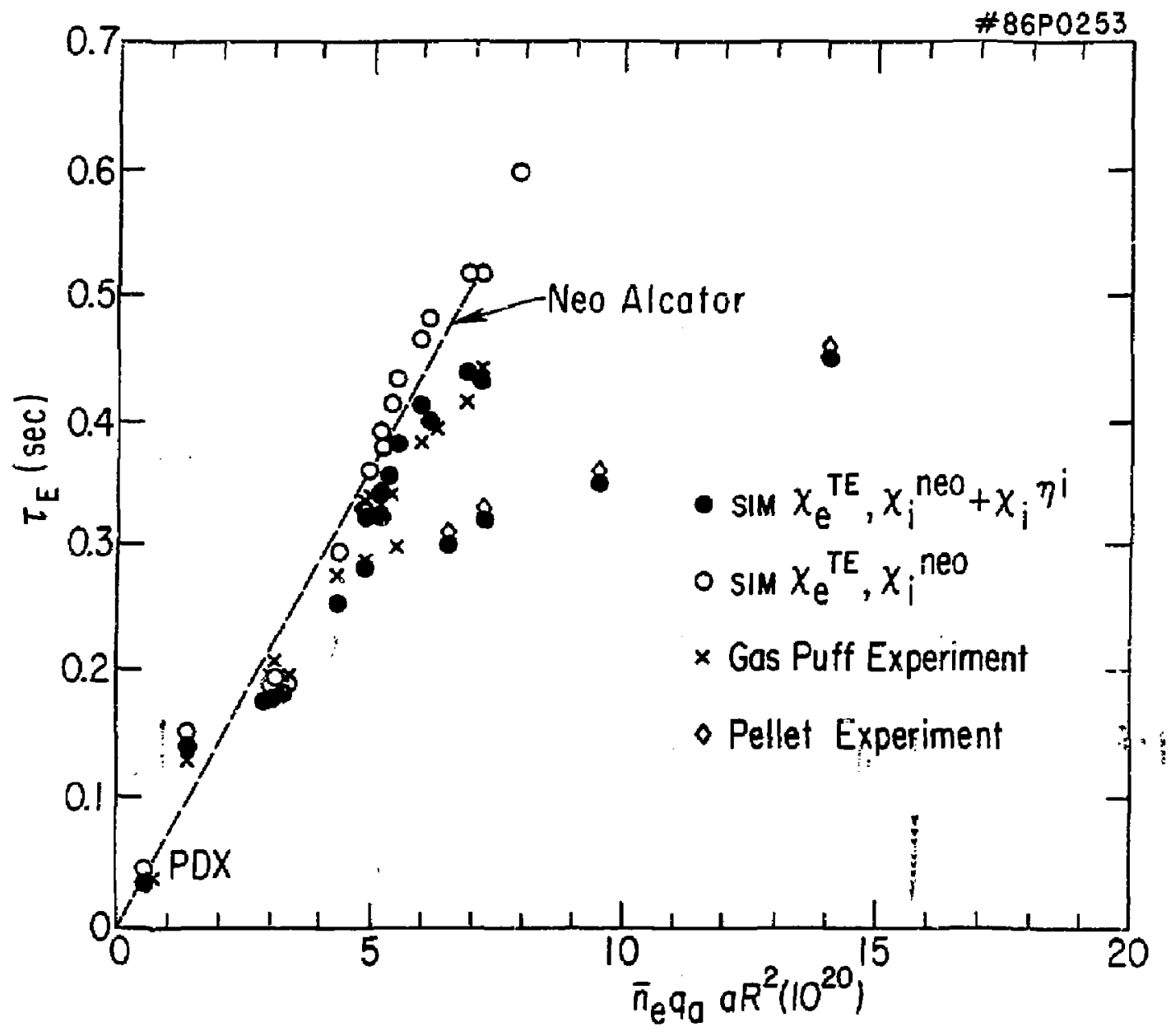

Fig. 1 
Or, Frank J. Paolonk, Ualv of wallongong, AUSTaAliA

Prof. M.H, Brannan, Univ Sycney, AUSTRALiA

Plasma Research Lab., Australlan Nat, Univ,, AUSTRALIA

Prof. I.R. Jones, Fll nders UniY., AUSTRALIA

Prof. F. Cap, Inst Theo Phys, AUSTRIA

Prof. M. Helndler, Institut fur Theoretische Physlk, AustalA

M. Goossens, Astronomisch Instltuut, GELGIUM

Ecole Rayale mliltaire, Lab de Phys Plasmas, BELGIUM

Com. of Eurapesn, Dg XII Fusion Frog, BELGIUly

Frof. R. Bovelque, Laberatoris voor Natuurkunde, BELGIUM

Dr. P.H. Sakonaks, Univ Estaduat, BRMZIL

Instituto De Posquisas Espociasi-INPE, ERAZIL

LIbrary, Atomlc Energy of Canade Limited, CNMADA

Or. M.P. Bachyrskl, MPB Tachnologles, InC., CNMAOA

Dr. H.M. Skersgard, Unlv of Saskatchewan, CANMA

ar. H. Barnard, Univarsity at Gritish Columbla, CANAOA

Prof. J. Tolehmann, Univ, of Montreal, CNNDA

Prof. S.R. Sreenlyssan, Un!versliy of Calgary, CAMADA

Prof. Tudor W. Johnston, INRS-Energle, CANADA

Dr. C.R. James, Unly. of Albarta, CANADA

Dr. Pater Lukar, Komensketho Unir, GZEChosLOVAKIA

The LIbrarfan, Culhen Laboratory, ENGLAND

Mrs, S.A. Hyteninson, JET Llorary. ENGLANO

c. Mouttot, Lab. de Physlave des Mllieux lonisés, FRNACE

J. Radat, CEN/CNDARACHE - Bat 506, FRAKCE

or. Tom Ual, Acodeny Blbljographle, HONG KOAG

Preprint Library, Cent Res Inst. Fhys, HUKGRY

Or. B. Desgupta, Saha Inst, INDilA

Dr. R.K. Chhajlanl, VIkron Univ. IRolA

Dr. P. Kar, Instil tute for Plasea Research, INDIA

Dr. PhIIIIp Rcsenau, I srael Inat Tech, ISRAEL

Prot. S. Cuperman, Tol Aviv UnIversity, ISRAEL

Librarlan, int'l Ctr theo Phys, ITALY

Próf. G. RostognI, UnIY DI Padove, ITALY

Miss Clella De Pa:o. Assoc EURATOM-ENEA, ITALY

Blblloteca, del ONR EllRatom, ItAly

Dr. H. Yando, Toshiba Res L Dev, JAPAN

Prot, I. Kougaal, Atomle Energy Ros. Ingtitute, JAPAN

Prot. KyojI Nishtkawa, Univ of HIroshias, JAPAN

DIrec. Dept. Lg. Tokemak Ros, JAER!, JAPAH

Prof, Satoshl Itoh, Kyushu Universlty, JAPAW

Research Info Conter, Nagoya UnIvarzlty, JAPAN

Prot. S. Tanaka, Kyoto Unlversity, JAPAY

Llorary, Kyoto Unlverslty, JAPAN

Prof. Nobuyukl Inoue, University of Tokyo, JAPAN

5, Mori, JAERI, JAPAN

M.H. KIm, Korea Advanced Enorgy Roswarch Instltute, KOREA

Prof. D.1. Chol, Adv. Inst 5cl \& Tech, KOREA

Prof, B.S. LIITy, UnI versity of Walkato, NEW ZEALANO

institute of Plasing Physles, PEOPLE'S REPUBLIC OF CHFNA

Librarlan. Institute of Phys., PEOPLE's REPUelic of CHINh

Library, tsing Hus University, PEOPLE'S REvBlic of CHINA
2. LI, Southwast inst. Physles, PEOPLE'S RePual tC OF CHIAA Prot. J.A.C. Cabral, Inst Superior Teen, PORTUGAL

Dr. Octavian Petrus, AL I CJZA University, ROMANIA

Dr. Jonan de vililers, Plesme Physlas, AEC, SO AFRICA

Prof. M.A. Hellberg, Universlty of Natal, SO AFRICA

Fusion DIV. Library, JEN, SPAIN

Dr. Lennert Stenflo, Unlversity of LAEA, SHEDEN

Llorary, Royal Inst Tech, SWEDEN

Prot. Hans WIInelmon, Chalmars Unly Tach, SWEDEN

Cantre Phys des Plasmas, Ecole Polytech fed, SwITZERLANo

Olbllotheat, Fon-l nst Yoor Plasns-Fys lca, THE NETHERLANDS

Dr. D.D. Ryitor, Slberlan Acad Sel, USSR

Or. G.A. El lsere, Kurchatov institute, US5R

Dr. V.A. Glukhlkh, Inst Electro-Physledl, USSR

Or. V.T. Tolck, Inst. Phys. Tech. USSA

Or. L.M. Kovrlzhnykh. Instltute Gen. Physlics, USSR

prof. T.d.M. Bayd, univ College N walos, Wales

Nucleor Res. Establishment, Julleh Ltd., W. GERHANY

Glbllathek, Inst, Fur Plasmaforschung, W. GERMANY

Dr. K. SehIndier, Ruhr Unlyarsitat, W. GERMANY

ASOEX Roading Rm, IPP/Max-Planck-Institut fur

Plasmaphyslk, W. GERMANY

Lrbrarlan, Haxtlanck InstItut, W. GERMANY

Prot. R.K. Janey, Inst Phys, Yugoslavia 